\title{
Nhân tố ảnh hưởng đến quyết định sử dụng dịch vụ Smartbanking- Nghiên cứu thực nghiệm tại BIDV - Chi nhánh Bắc Sài gòn
}

\author{
Hà Nam Khánh Giao \\ Khoa Vận tải Hàng không, Học viện Hàng không Việt Nam \\ Trần Kim Châu \\ Ngân hàng TMCP Đầu tư và Phát triển Việt Nam, Chi nhánh Ba Mươi Tháng Tư
}

Nghiên cưu nhằm xác định và đo lường các nhân tố ảnh hưởng đến quyết định sủ dụng dịch vu smart banking tại Ngân hàng Thương mại cồ phần Đầu tu và Phát triển Việt Nam-Chi nhánh Bắc Sài Gòn (BIDV BSG), bằng việc khảo sát 235 khách hàng cá nhân của Chi nhánh. Nghiên cứu sử dụng công cu SPSS 20 để phân tích độ tin cậy thang đo qua hệ số Cronbach's Alpha, phân tích nhân tố khám phá EFA, mô hình hồi quy tuyến tính bội. Kết quả nghiên cưu cho thấy các nhân tố tác động tích cực, sắp xếp theo độ mạnh giảm dần, bao gồm: Cảm nhận dễ sủ dụng, Cảm nhận sụ hưu ích, Cảm nhận sụ tin tưởng tới quyết định sủ dụng Smart banking của khách

\section{The factors affect on the Decision of using Smart Banking service at Bank of Investment and Development of Viet Nam- North Saigon Branch}

Abstract: This study aims at determining and measuring the factors affect on the decision of using smart banking at Bank of Investment and Development of Viet Nam- North Saigon Branch (BIDV BSG), by surveying 235 personal customers of the branch. The study uses SPSS 20 tool to analyze the scale credibility through Cronbach's Alpha, exploratory factor analyze, and multiple linear regression analyze.

The results show that there are positively impacted factors, arranged by decreasing impact: Perceived Ease of Use, Perceived Usefulness, Perceived Credibility to decision of using smart banking of customers. Meanswhile, there are negatively impacted factors, arranged by decreasing impact: Perceived Risks, Perceived Cost. The results also help management to recognize the importance of the factors which effect on decision of usinf smart banking of the personal customers, from there, management can adopt the strategies and tactics reasonably in the context of fierce competition nowadays.

Keywords: The factors, Decision of using smart banking, Bank of Investment and Development of Viet NamNorth Saigon Branch

\section{Giao Nam Khanh Ha}

Email: khanhgiaohn@yahoo.com

Faculty of Air Transport, Vietnam Aviation Academy

\section{Chau Kim Tran}

Email: chautk@bidv.com.vn

Bank of Investment and Development of Viet Nam- 30 April Banch 
hàng. Trong khi đó, các nhân tố tác động tiêu cực, sắp xếp theo độ mạnh giảm dần, bao gồm: Cảm nhận về rủi ro, Cảm nhân về chi phí. Kết quả cũng giúp cho các nhà quản trị nhận thấy được tầm quan trọng của các nhân tố ảnh huởng đến quyết định sử dụng của khách hàng cá nhân, và tù đó có những điều chỉnh chiến luợc và hành động phù hợp trong quá trình cạnh tranh khốc liệt hiện nay đối với các ngân hàng.

Tù khóa: Nhân tố ảnh hưởng, Quyết định sử dụng, dịch vu smart banking, Ngân hàng Thương mai cổ phần Đầu tu và Phát triển Việt Nam-Chi nhánh Bắc Sài gòn (BIDV BSG).

\section{Giới thiệu}

Theo Báo cáo cuối tháng 6/2019 của Bộ Thông tin \& Truyền thông, đã có 134,5 triệu thuê bao di động, trong đó có 121,12 triệu thuê bao (90\%) sử dụng điện thoại thông minh (smartphone). Đây là tín hiệu đáng mừng khi mà các ngân hàng sử dụng các ứng dụng để quản lý thông tin tài khoản khách hàng dễ dàng hơn thông qua điện thoại.

Trong môi trường toàn cầu, ngành Ngân hàng là một trong những lĩnh vực cần hiện đại hóa và hội nhập nhanh nhất để đáp ứng những nhu cầu tăng cao của khách hàng. Một trong những ứng dụng được sử dụng phổ biến là ứng dụng dịch vụ Smart Banking của các ngân hàng. Đây là dịch vụ ngân hàng trên điện thoại di động thông minh, cho phép khách hàng cá nhân thực hiện các giao dịch tài chính, phi tài chính và các tiện ích nâng cao do ngân hàng cung cấp (Hughes, 2018). Dịch vụ được cung cấp đến tất cả các khách hàng sử dụng điện thoại thông minh dùng hệ điều hành Android, IOS, Window Phone. Dịch vụ này không những đem đến những tiện ích cho cuộc sống hiện đại ngày nay như chuyển tiền, thanh toán, dịch vụ thẻ, mua sắm, tiền gửi online, nạp tiền điện thoại... mà còn bảo mật được thông tin khách hàng nhờ ứng dụng mở khóa bằng vân tay, face ID.

Các tính năng của dịch vụ Smart Banking hiện các ngân hàng cung cấp gồm: Vấn tin số dư tài khoản của khách hàng; Truy vấn chi tiết thông tin thẻ, lịch sử giao dịch, sao kê của thẻ ghi nợ nội địa, thẻ ghi nợ quốc tế và thẻ tín dụng quốc tế; Đăng ký sản phẩm dịch vụ online (tiền vay, thẻ); Tìm kiếm ATM/Chi nhánh; Tra cứu thông tin tỷ giá, lãi suất; Chuyển tiền thanh toán, chuyển tiền liên ngân hàng 24/7; Thanh toán trực tuyến: nạp tiền điện thoại, mua mã thẻ cào, thanh toán hóa đơn tiền điện, nạp tiền học phí, thanh toán vé máy bay, học phí, truyền hình, viễn thông, nạp tiền ví điện tử, nạp tiền game; Các tiện ích nâng cao: mua vé máy bay qua đại lý VNPAY, quản lý đầu tư, trao đồi thông tin, thư giãn giải trí... (Hughes, 2018).

BIDV đang là ngân hàng bán lẻ tốt nhất trong 05 năm qua do The Asian Banker bình chọn (TTBC số 3/2019) và Chi nhánh Bắc Sài Gòn là chi nhánh cấp một trực thuộc BIDV, tiền thân là Chi nhánh Tân Bình. Về lợi nhuận trước thuế, BIDV BSG đứng thứ hai trên địa bàn TP. Hồ Chí Minh, đứng thứ 15 trên toàn hệ thống BIDV. BIDV là một trong các ngân hàng đang triển khai mạnh mẽ dịch vụ smart banking tới các khách 
hàng cá nhân. Vậy lý do nào để khách hàng lựa chọn và quyết định sử dụng dịch vụ smart banking tại các ngân hàng?

Hiện đã có nhiều nghiên cứu trong và ngoài nước về dịch vụ (Hà Nam Khánh Giao, 2004, 2018; Hà Nam Khánh Giao, Ao Thu Hoài và Phạm Quang Vinh, 2019), nhưng chỉ một số tác giả (Luarn và Lin, 2005; Wu và Wang, 2005; Nguyễn Thế Phương, 2014; Lê Tô Minh Tân, 2013) đã quan tâm đến dịch vụ smart banking, mobile banking, và các nhân tố tác động đến quyết định sử dụng smart banking, mobile banking trên thế giới và tại Việt Nam. Tuy nhiên, cũng chưa có nghiên cứu sâu tại BIDV về vấn đề này. Việc nghiên cứu quyết định sử dụng dịch vụ smart banking tại BIDV BSG là cần thiết, là gợi ý cho các nhà quản lý tại BIDV BSG nói riêng, các ngân hàng nói chung khi xây dựng chiến lược khách hàng trong bối cảnh cạnh tranh dịch vụ ngân hàng đang gia tăng như hiện nay.

\section{Tổng quan lý thuyết}

\subsection{Các mô hình lý thuyết đánh giá hành vi chấp nhận sử dụng dịch vụ}

\section{Mô hình chấp nhận công nghệ} (Technology Acceptance Model- TAM) (Davis 1989; Davis và ctg, 1989) đã được thừa nhận và áp dụng rộng rãi để kiểm tra mức độ chấp nhận của người sử dụng đối với các ứng dụng hệ thống công nghệ thông tin. Mô hình TAM bao gồm hai cấu trúc: (1) Cảm nhận sụ hũu ích (Perceived usefulness): mức độ mà một người tin rằng việc sử dụng một hệ thống, một dịch vụ hay sản phẩm công nghệ đặc biệt sẽ nâng cao hiệu suất công việc của họ; và (2) cảm $n h a ̂ ̣ n$ dê sử dụng (Perceived ease of use): mức độ mà một người tin rằng sử dụng một hệ thống, một dịch vụ hay sản phẩm công nghệ mới cụ thể họ cũng không khó khăn để học cách sử dụng nó, việc sử dụng sẽ đơn giản và dễ hiểu. Trong đó, nhân tố dễ sử dụng có tác động đến cảm nhận về sự hữu ích.

\section{Mô hình chấp nhận công nghệ mở rộng} (Extended TAM): Luarn và Lin (2004) đã mở rộng mô hình TAM ban đầu bằng cách thêm một số nhân tố có liên quan nhằm tìm hiểu quyết định sử dụng dịch vụ Mobile Banking (là một bộ phận của Smart Banking) tại Đài Loan: Cảm nhận sụ tin tương (Perceived credibility), Cảm nhận về chi phí (Perceived cost), Cảm nhận về tụ tin (Perceived self-efficacy).

Wu và Wang (2005) dựa trên mô hình Extended TAM thêm vào: Cảm nhận rủi ro và chi phí tài chính. Gần đây, Jeong và Yoon (2013) cũng dựa trên mô hình TAM mở rộng, phân tích 5 cảm nhận của người sử dụng ảnh hưởng đến quyết định sử dụng mobile banking tại Singapore ( sự hữu ích, dễ sử dụng, sự tín nhiệm, tự cảm nhận hiệu quả và chi phi tài chính), với kết quả: cảm nhận sự hữu ích có ảnh hưởng nhất, nhưng cảm nhận chi phí tài chính thì không có ảnh hưởng.

Tại Việt Nam, nghiên cứu của Nguyễn Thế Phương (2014) đã sử dụng mô hình TAM mở rộng làm cơ sở lý thuyết để điều tra quyết định sử dụng dịch vụ Mobile Banking tại thành phố Hồ Chí Minh. Kết quả cho thấy: Tính hữu ích, dễ dàng sử dụng, sự tin tưởng, chi phí và rủi ro sử dụng có tác động quan trọng đến quyết định sử dụng dịch vụ Mobile Banking của khách hàng tại địa bàn này. Tương tự, Lê Tô Minh Tân (2013) nghiên cứu quyết định sử dụng dịch vụ ngân hàng trực tuyến của khách hàng cá nhân ở Thừa Thiên Huế, kết quả cho thấy các nhân tố như cảm nhận rủi ro, cảm 
nhận dễ sử dụng, cảm nhận sự hữu ích có tác động đến quyết định sử dụng kênh ngân hàng trực tuyến, trong đó, cảm nhận sự hữu ích có tác động lớn nhất.

\section{Lýthuyết khuếch tán đổi mới (Innovations} Diffusion Theory- IDT) được đưa ra bởi Roger (1995) để giải thích quá trình chấp nhận các ý tưởng mới, công nghệ mới của người sử dụng. Quá trình chấp nhận những tác động đổi mới bao gồm 5 giai đoạn: (1) giai đoạn nhận thức; (2) giai đoạn thuyết phục; (3) giai đoạn đưa ra quyết định; (4) giai đoạn thực hiện; và (5) giai đoạn xác nhận.

Lý thuyết hợp nhất về chấp nhận và sử dụng công nghệ (UTAUT) được phát triển bởi Venkatesh và ctg (2003). Mô hình UTAUT là mô hình hợp nhất từ tám mô hình chấp nhận công nghệ trước đó, mô hình này cho rằng có 4 nhân tố: mong đợi về thành tích (Performance Expectancy), mong đợi về sự nỗ lực (Effort Expectancy), ảnh hưởng xã hội (Social Influence) và điều kiện thuận tiện (Facilitating Conditions) là những nhân tố ảnh hưởng trực tiếp đến quyết định sử dụng và hành vi sử dụng. Trong khi đó, giới tính, tuổi, kinh nghiệm và sự tự nguyện được cho là có tác động gián tiếp đến 4 nhân tố chính phía trên (Venkatesh và ctg, 2003).

Kết quả nghiên cứu của Yu (2012) cho thấy: chi phí tài chính, ảnh hưởng của xã hội và sự tin tưởng có ảnh hưởng mạnh đến quyết định sử dụng mobile banking. Trong khi đó, kết quả nghiên cứu của Foon và Fah (2011) cho thấy: kết quả kỳ vọng, nỗ lực kỳ vọng, ảnh hưởng xã hội, các điều kiện thuận tiện và sự tin tưởng đều đóng vai trò quan trọng đến quyết định sử dụng internet banking (trung bình có thể giải thích 56\% sự thay đổi quyết định sử dụng dịch vụ này).

\subsection{Mô hình nghiên cứu đề xuất}

Các mô hình nghiên cứu gần đây về smart banking áp dụng thành công ở trong và ngoài nước phần lớn đều xuất phát từ mô hình TAM của Davis (1989) và mô hình TAM mở rộng (Extended TAM) của Luarn và Lin (2005). Do đó, nhóm tác giả đã đề

Hình 1. Mô hình nghiên cứu đề xuất

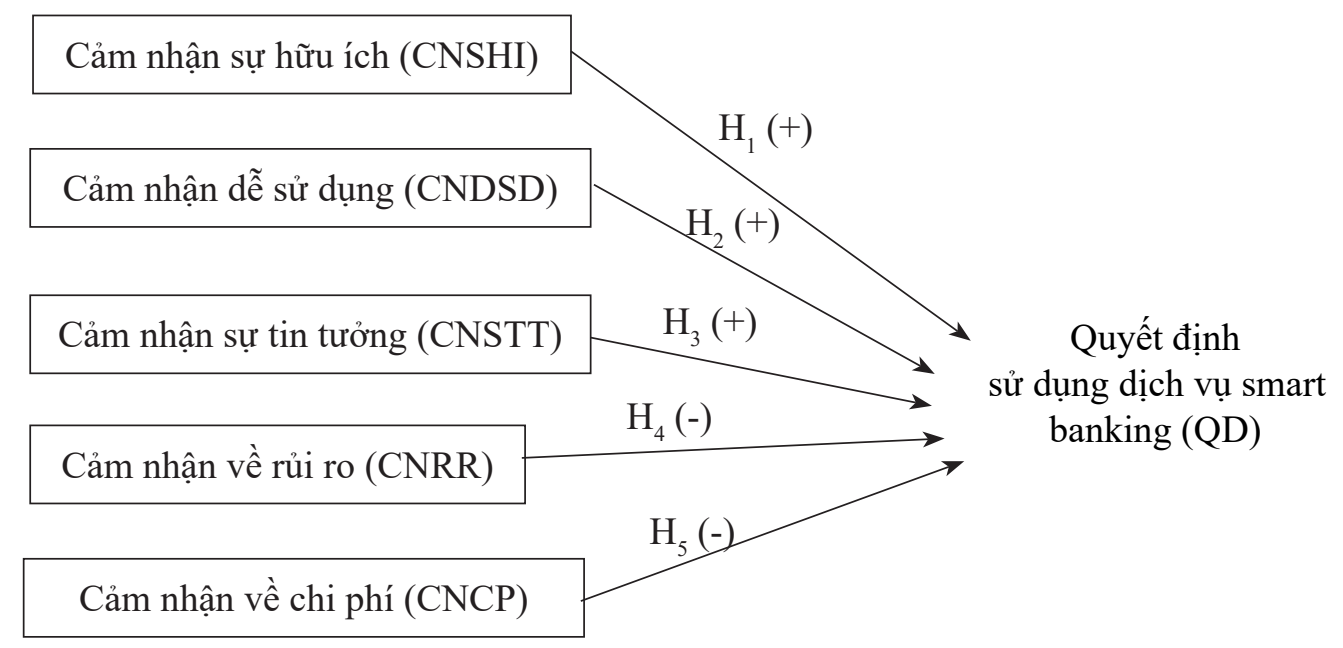

Nguồn: Nhóm tác giả đề xuất dụa trên mô hình TAM mở rộng và các nghiên cứu trong nước 
xuất sử dụng mô hình TAM mở rộng, có chọn lọc và bổ sung thêm một số nhân tố phù hợp với điều kiện của Việt Nam từ nghiên cứu của Nguyễn Thế Phương (2014) và Lê Tô Minh Tân (2013) (Bảng 1). Từ đó, kết hợp với khảo lược các nghiên cứu có liên quan, nhóm tác giả đề xuất mô hình nghiên cứu như trong Hình 1 , cùng các giả thuyết:

$\mathrm{H}_{1}$ : Cảm nhận sự hữu ích có ảnh hưởng cùng chiều đến quyết định sử dụng dịch vụ Smart banking.

$\mathrm{H}_{2}$ : Cảm nhận dễ sử dụng ảnh hưởng cùng chiều đến quyết định sử dụng dịch vụ Smart banking.

$\mathrm{H}_{3}$ : Cảm nhận sự tin tưởng ảnh hưởng cùng chiều đến quyết định sử dụng dịch vụ Smart banking.

$\mathrm{H}_{4}$ : Cảm nhận về chi phí có tác động ngược chiều đến quyết định sử dụng dịch vụ Smart banking.

$\mathrm{H}_{5}$ : Cảm nhận về rủi ro có tác động ngược chiều đến quyết định sử dụng dịch vụ Smart banking.

\section{Phương pháp nghiên cứu}

Sau khi khảo lược lý thuyết và các công trình nghiên cứu của các tác giả trong và ngoài nước nhằm tìm hiểu các nhân tố ảnh hưởng đến quyết định sử dụng dịch vụ smart banking tại BIDV BSG, nhóm tác giả xây dựng mô hình nghiên cứu đề xuất (Hình 1) và giả thuyết nghiên cứu. Tiếp theo, nhóm tác giả phỏng vấn chuyên gia- là các chuyên viên smart banking và mobile banking của BIDV BSG, đồng thời cũng thực hiện cuộc phỏng vấn nhóm (focus group) đối với 8 khách hàng có sử dụng smart banking của
Chi nhánh. Kết quả của phỏng vấn chuyên gia và phỏng vấn nhóm được sử dụng để thiết kế nội dung thông tin khảo sát. Ngoài ra, Bảng khảo sát sơ bộ còn được phỏng vấn thử (pilot test) với 50 khách hàng có sử dụng smart banking tại BIDV BSG. Kết quả phỏng vấn chuyên gia, phỏng vấn nhóm, khảo sát sơ bộ được dùng vào việc hình thành các phát biểu chính thức trong Bảng câu hỏi khảo sát để thu thập thông tin phục vụ cho kiểm định và đánh giá mô hình nghiên cứu, và không thay đổi so với nội dung các biến quan sát trong Bảng 1 .

Đối tượng khảo sát là những người đã/đang sử dụng smart banking tại BIDV BSG. Do hạn chế về thời gian và không gian, khảo sát chính thức chỉ được tiến hành theo phương pháp chọn mẫu thuận tiện tại Chi nhánh từ 15/10/2019-31/12/2019. Nhóm tác giả thực hiện phỏng vấn và phát bảng hỏi trực tiếp với khách hàng đến giao dịch tại một số phòng giao dịch của Chi nhánh. 250 bảng câu hỏi được phát ra, trả về 235 kết quả trả lời hợp lệ. Kích thước mẫu đạt yêu cầu theo tiêu chuẩn của Hà Nam Khánh Giao \& Bùi Nhất Vương (2019) là lớn hơn 5 lần số lượng biến quan sát (tối thiểu 140 phiếu).

Để đo lường thái độ, mức cảm nhận của đối tượng tham gia khảo sát, các biến quan sát được đo lường bằng thang đo Likert với 5 mức độ phổ biến sau: (1) Rất không đồng ý; (2) không đồng ý; (3) bình thường; (4) đồng ý; (5) rất đồng ý. Toàn bộ mẫu hợp lệ được xử lý dữ liệu bằng phần mềm SPSS 20.0 để tiến hành các bước phân tích độ tin cậy, phân tích tương quan, phân tích nhân tố, phân tích hồi quy và kiểm định giả thuyết.

\section{Kết quả nghiên cứu và thảo luận}

\section{1. Đặc điểm mẫu khảo sát}


Nhân tố ảnh hưởng đến quyết định sử dụng dịch vụ Smart Banking- Nghiên cứu thực nghiệm tại BIDV-Chi nhánh Bắc Sài Gòn

Bảng 2 cho thấy các đặc trưng của mẫu nghiên cứu.

Mẫu khảo sát có 162 người là nam (chiếm 68,9\%), độ tuổi 41-50 chiếm cao nhất trong các nhóm (75 người, 31,9\%); nghề nghiệp chủ yếu là nhân viên văn phòng (74 người, chiếm $31,5 \%$ ) và kinh doanh tự do (69 người chiếm 29,4\%). Nhóm người có thu nhập từ 9- 15 triệu đồng có 71 (chiếm $30,2 \%$ ), thu nhập từ $15-20$ triệu đồng có 68 (chiếm 28,9\%) là nhóm có thu nhập chiếm cao nhất. Trình độ đại học có 72 người (chiếm 30,6\%) và có 70 người có trình độ học vấn sau đại học (chiếm 29,8\%) là 2 nhóm có trình độ cao nhất. Thời gian sử dụng dịch vụ BIDV cũng khá cao: 90 người từ 2-5 năm (chiếm 38,3\%), 84 người (chiếm $35,7 \%$ ) trên 5 năm. Dù phương pháp chọn mẫu thuận tiện, việc phân bố mẫu khá hợp lý, cho thấy khả năng có được độ tin cậy cần thiết (Hà Nam Khánh Giao \& Bùi Nhất Vương, 2019).

Ngoài Ngân hàng BIDV, có 111 người sử dụng dịch vụ của ngân hàng Vietcombank

Bảng 1. Các biến quan sát trong mô hình nghiên cứu

\begin{tabular}{|c|c|c|c|}
\hline \multicolumn{2}{|c|}{ Biến quan sát } & Thang đo & Nguồn \\
\hline \multicolumn{4}{|c|}{ Cảm nhận sự hữu ích (H1) } \\
\hline CNSHI1 & $\begin{array}{l}\text { Giao dịch qua Smart banking rất nhanh chóng, không } \\
\text { phải mất thời gian đến ngân hàng }\end{array}$ & \multirow{5}{*}{ Likert 1-5 } & \multirow{5}{*}{$\begin{array}{l}\text { Luarn và Lin, } \\
2005 \\
\text { Wu và Wang, } \\
\text { 2005; } \\
\text { Nguyễn Thế } \\
\text { Phương, 2014, } \\
\text { Lê Tô Minh } \\
\text { Tân, } 2013\end{array}$} \\
\hline CNSHI2 & $\begin{array}{l}\text { Smart banking giúp tôi có thể thực hiện giao dịch ngân } \\
\text { hàng bất cứ khi nào }(24 / 24 \mathrm{~h})\end{array}$ & & \\
\hline CNSHI3 & $\begin{array}{l}\text { Sử dụng Smart banking giúp tôi thực hiện các giao dịch } \\
\text { ngân hàng dễ dàng hơn so với giao dịch tại quầy }\end{array}$ & & \\
\hline CNSHI4 & Tôi cảm thấy tiện lợi hơn khi sử dụng Smart banking & & \\
\hline CNSHI5 & $\begin{array}{l}\text { Giao dịch qua Smart banking giúp tôi tiết kiệm chi phí } \\
\text { hơn so với hình thức thông thường }\end{array}$ & & \\
\hline \multicolumn{4}{|c|}{ Cảm nhận dễ sử dụng (H2) } \\
\hline CNDSD1 & Học sử dụng dịch vụ Smart banking rất dễ dàng & \multirow{4}{*}{ Likert 1-5 } & \multirow{4}{*}{$\begin{array}{l}\text { Luarn và Lin, } \\
2005 \\
\text { Wu và Wang, } \\
\text { 2005; } \\
\text { Nguyễn Thế } \\
\text { Phương, 2014; } \\
\text { Lê Tô Minh } \\
\text { Tân, } 2013 \\
\end{array}$} \\
\hline CNDSD2 & Thực hiện các giao dịch Smart banking rất dễ dàng & & \\
\hline CNDSD3 & $\begin{array}{l}\text { Các hướng dẫn khi giao dịch Smart banking rất rõ ràng } \\
\text { và dễ hiểu }\end{array}$ & & \\
\hline CNDSD4 & Nhìn chung tôi thấy Smart banking rất dễ sử dụng & & \\
\hline \multicolumn{4}{|c|}{ Cảm nhận sự tin tưởng (H3) } \\
\hline CNSTT1 & $\begin{array}{l}\text { Tôi tin rằng thông tin giao dịch của tôi được giữ bí mật } \\
\text { khi sử dụng dịch vụ Smart banking }\end{array}$ & \multirow{5}{*}{ Likert 1-5 } & \multirow{5}{*}{$\begin{array}{l}\text { Luarn và Lin, } \\
2005 \\
\text { Wu và Wang, } \\
\text { 2005; } \\
\text { Nguyễn Thế } \\
\text { Phương, } 2014\end{array}$} \\
\hline CNSTT2 & $\begin{array}{l}\text { Tôi tin rằng giao dịch qua Smart banking cũng an toàn } \\
\text { như giao dịch qua quầy tại ngân hàng }\end{array}$ & & \\
\hline CNSTT3 & $\begin{array}{l}\text { Tôi tin rằng Smart banking có thể bảo mật các thông tin } \\
\text { tài chính cá nhân của tôi }\end{array}$ & & \\
\hline CNSTT4 & $\begin{array}{l}\text { Tôi tin rằng sử dụng Smart banking rất đáng tin cậy cho } \\
\text { các giao dịch tài chính }\end{array}$ & & \\
\hline CNSTT5 & $\begin{array}{l}\text { Tôi tin rằng ngân hàng sẽ trung thực với tôi trong các } \\
\text { giao dịch qua Smart banking }\end{array}$ & & \\
\hline
\end{tabular}




\begin{tabular}{|c|c|c|c|}
\hline \multicolumn{2}{|c|}{ Biến quan sát } & Thang đo & Nguồn \\
\hline \multicolumn{4}{|c|}{ Cảm nhận về chi phí (H4) } \\
\hline CNCP1 & $\begin{array}{l}\text { Chi phí sử dụng Smart banking là khoản chi lớn đối với } \\
\text { tôi (Phí hàng tháng hay phí khi thực hiện giao dịch) }\end{array}$ & \multirow{4}{*}{ Likert 1-5 } & \multirow{4}{*}{$\begin{array}{l}\text { Luarn và Lin, } \\
2005 \\
\text { Wu và Wang, } \\
\text { 2005; } \\
\text { Nguyễn Thế } \\
\text { Phương, } 2014\end{array}$} \\
\hline CNCP2 & $\begin{array}{l}\text { Chi phí kết nối phải trả cho nhà mạng (4G, SMS) là quá } \\
\text { đắt khi sử dụng dịch vụ Smart banking }\end{array}$ & & \\
\hline CNCP3 & $\begin{array}{l}\text { Chi phí để cài đặt ứng dụng Smart banking trên điện } \\
\text { thoại là đắt tiền đối với tôi }\end{array}$ & & \\
\hline CNCP4 & $\begin{array}{l}\text { Nhìn chung sử dụng Smart banking tốn chi phí giao dịch } \\
\text { nhiều hơn so với các kênh giao dịch khác (giao dịch tại } \\
\text { quầy, giao dịch qua internet...) }\end{array}$ & & \\
\hline \multicolumn{4}{|c|}{ Cảm nhận rủi ro (H5) } \\
\hline CNRR1 & $\begin{array}{l}\text { Tôi e ngại nếu giao dịch qua Smart banking bị lỗi tôi có } \\
\text { thể mất tiền trong tài khoản }\end{array}$ & \multirow{5}{*}{ Likert 1-5 } & \multirow{5}{*}{$\begin{array}{l}\text { Luarn và Lin, } \\
2005 \\
\text { Wu và Wang, } \\
\text { 2005; } \\
\text { Nguyễn Thế } \\
\text { Phương, 2014; } \\
\text { Lê Tô Minh } \\
\text { Tân, } 2013\end{array}$} \\
\hline CNRR2 & $\begin{array}{l}\text { Tôi e ngại rằng việc cung cấp thông tin cá nhân cho các } \\
\text { giao dịch qua Smart banking là không an toàn }\end{array}$ & & \\
\hline CNRR3 & $\begin{array}{l}\text { Tôi e ngại việc sử dụng Smart banking có thể bị kẻ xấu } \\
\text { đánh cắp và sử dụng tài khoản của tôi }\end{array}$ & & \\
\hline CNRR4 & $\begin{array}{l}\text { Tôi e ngại nếu bị mất điện thoại khi sử dụng Smart } \\
\text { banking thì tiền của tôi cũng sẽ bị mất }\end{array}$ & & \\
\hline CNRR5 & $\begin{array}{l}\text { Tôi e ngại rằng khi tôi gặp những vấn đề trên Smart } \\
\text { banking mà ngân hàng không giải quyết thỏa đáng cho } \\
\text { tôi }\end{array}$ & & \\
\hline \multicolumn{4}{|c|}{ Quyết định sử dụng dịch vụ Smart Banking } \\
\hline QD1 & $\begin{array}{l}\text { Tôi có động lực mạnh mẽ để sử dụng dịch vụ Smart } \\
\text { Banking của BIDV }\end{array}$ & \multirow{3}{*}{ Likert 1-5 } & \multirow{3}{*}{$\begin{array}{l}\text { Luarn và Lin, } \\
2005 \\
\text { Wu và Wang, } \\
\text { 2005; } \\
\text { Nguyễn Thế } \\
\text { Phương, } 2014\end{array}$} \\
\hline QD2 & Tôi sẽ sử dụng dịch vụ Smart Banking của BIDV & & \\
\hline QD3 & $\begin{array}{l}\text { Tôi sẽ giới thiệu dịch vụ Smart Banking của BIDV cho } \\
\text { người khác }\end{array}$ & & \\
\hline
\end{tabular}

Nguồn: Đề xuất của nhóm tác giả tù̀ tổng quan nghiên cứu, 2020

Bảng 2. Kết quả thống kê mô tả dữ liệu mẫu nghiên cứu

\begin{tabular}{|c|c|c|c|}
\hline Thống kê mẫu & Nội dung & Tần suất & Tỷ lệ (\%) \\
\hline \multirow{2}{*}{ Giới tính } & Nam & 162 & 68,9 \\
\hline & Nữ & 73 & 31,1 \\
\hline \multirow{4}{*}{ Độ tuổi } & $18-30$ & 24 & 10,2 \\
\hline & $31-40$ & 63 & 26,8 \\
\hline & $41-50$ & 75 & 31,9 \\
\hline & $>50$ & 73 & 31,1 \\
\hline \multirow{4}{*}{ Nghề nghiệp } & Học sinh/ sinh viên & 33 & 14,0 \\
\hline & Công nhân & 59 & 25,1 \\
\hline & Nhân viên văn phòng & 74 & 31,5 \\
\hline & Kinh doanh tự do & 69 & 29,4 \\
\hline
\end{tabular}


Nhân tố ảnh hưởng đến quyết định sử dụng dịch vụ Smart Banking- Nghiên cứu thực nghiệm tại BIDV- Chi nhánh Bắc Sài Gòn

\begin{tabular}{|c|c|c|c|}
\hline Thống kê mẫu & Nội dung & Tần suất & Tỷ lệ (\%) \\
\hline \multirow{5}{*}{ Thu nhập (triệu đồng) } & $<5$ & 24 & 10,2 \\
\hline & $5-9$ & 60 & 25,5 \\
\hline & $9-15$ & 71 & 30,2 \\
\hline & $15-20$ & 68 & 28,9 \\
\hline & $>20$ & 12 & 5,1 \\
\hline \multirow{4}{*}{ Trình độ học vấn } & Phổ thông & 32 & 13,6 \\
\hline & Cao đẳng & 61 & 26,0 \\
\hline & Đại học & 72 & 30,6 \\
\hline & Sau đại học & 70 & 29,8 \\
\hline \multirow{4}{*}{ Anh/chị sử dụng dịch vụ BIDV được bao lâu } & Dưới 1 năm & 22 & 9,4 \\
\hline & 1 -2 năm & 39 & 16,6 \\
\hline & 2 - 5 năm & 90 & 38,3 \\
\hline & Trên 5 năm & 84 & 35,7 \\
\hline \multirow{7}{*}{$\begin{array}{l}\text { Ngoài BIDV, anh/chị còn sử dụng dịch vụ của } \\
\text { ngân hàng nào }\end{array}$} & Vietcombank & 111 & 23,4 \\
\hline & Á Châu (ACB) & 95 & 20,0 \\
\hline & Đông Á & 92 & 19,4 \\
\hline & Techcombank & 52 & 10,9 \\
\hline & VP Bank & 52 & 10,9 \\
\hline & Sacombank & 39 & 8,2 \\
\hline & Viettinbank & 34 & 7,2 \\
\hline $\begin{array}{l}\text { Anh/chị đã từng nghe qua dịch vụ Smart } \\
\text { Banking của BIDV chưa }\end{array}$ & Đã từng nghe & 235 & 100,0 \\
\hline
\end{tabular}

Nguồn: Phân tích tù dũ liệu thu thập được bằng SPSS 20

(chiếm 23,4\%), 95 người sử dụng của ngân hàng $\mathrm{ACB}$ (chiếm 20\%), 92 người sử dụng ngân hàng Đông Á (chiếm 19,4\%), 52 người sử dụng ngân hàng Techcombank (chiếm 10,9\%), 52 người sử dụng ngân hàng VP Bank, 39 người sử dụng ngân hàng Sacombank (chiếm $8,2 \%$ ), và cuối cùng, có 34 người sử dụng ngân hàng Vietinbank (chiếm 7,4\%). Đây có thể là điều đáng quan tâm trong nghiên cứu này, do trong quá trình trả lời khảo sát, cảm nhận của đáp viên có thể bị ảnh hưởng bởi việc sử dụng đồng thời các dịch vụ từ các ngân hàng khác nhau, tuy nhiên là hữu ích khi đáp viên có sự so sánh cảm nhận đối với việc sử dụng dịch vụ từ các ngân hàng, như đã được chỉ ra trong kết quả của một số nghiên cứu gần đây (Ha Nam Khanh Giao, Bui Nhat Vuong và Tran Nhu Quan, 2019; Bui Nhat Vuong \& Ha Nam Khanh Giao, 2019).

\subsection{Kiểm định độ tin cậy thang đo bằng Cronchbach's alpha}

Kết quả tại Bảng 3 cho thấy, sau khi loại CNCP4, các thang đo đều có hệ số Cronbach's Alpha khá cao $(>0,7)$, hệ số tương quan biến- tổng lớn hơn 0,6 , do đó chúng đều được sử dụng cho phân tích EFA tiếp theo. 
Bảng 3. Hệ số Cronbach's Alpha

\begin{tabular}{lccc}
\hline Biến quan sát & Số biến quan sát & Cronbach's Alpha & $\begin{array}{c}\text { Hê số tương quan } \\
\text { biến- tống nhỏ nhất }\end{array}$ \\
\hline Cảm nhận sự hữu ích (CNSHI) & 5 & 0,915 & 0,742 \\
\hline Cảm nhận dễ sử dụng (CNDSD) & 4 & 0,808 & 0,600 \\
\hline Cảm nhận sự tin tưởng (CNSTT) & 5 & 0,868 & 0,661 \\
\hline Cảm nhận về chi phí (CNCP) & 3 & 0,794 & 0,606 \\
\hline Cảm nhận về rủi ro (CNRR) & 5 & 0,842 & 0,625 \\
\hline Quyết định sử dụng dịch vụ (QD) & 3 & 0,804 & 0,602 \\
\hline
\end{tabular}

Nguồn: Phân tích tù dũ liệu thu thập được bằng SPSS 20

\subsection{Phân tích EFA}

Kết quả EFA cho 22 biến quan sát của các biến độc lập ảnh hưởng đến Quyết định sử dụng smart banking tại BIDV BSG, hệ số $\mathrm{KMO}$ đạt 0,850 và mức ý nghĩa $(\mathrm{Sig}$. $=$ $0,000)$ của kiểm định Bartlett's là $1 \%$ cho thấy các biến này có tương quan với nhau và hoàn toàn phù hợp với phân tích nhân tố. Giá trị Eigen là 1,350 > 1, với phương sai trích là $68,033 \%(>50 \%)$ cho biết 5 nhân tố giải thích được $68,033 \%$ biến thiên các dữ liệu (Bảng 4).

Phân tích nhân tố biến phụ thuộc Quyết định sử dụng dịch vụ cho thấy hệ số KMO đạt 0,702 và mức ý nghĩa $($ Sig. $=0,000)$ của kiểm định Bartlett's là $1 \%$ cho thấy các biến này có tương quan với nhau và hoàn toàn phù hợp với phân tích nhân tố. Giá trị Eigen là 2,157> 1, ba (3) biến quan sát của biến phụ thuộc đã trích ra 1 nhân tố với tổng phương sai trích 71,902\%. Hệ số tải nhân tố của 3 biến quan sát này đều lớn hơn 0,7 .

\subsection{Ma trận hệ số tuơng quan}

Để xem xét mối quan hệ tương quan tuyến tính giữa các biến độc lập CNSHI, CNDSD, CNSTT, CNCP, CNRR và biến phụ thuộc $\mathrm{QD}$, kiểm định hệ số tương quan
Bảng 4. Bảng ma trận xoay nhân tố

\begin{tabular}{|c|c|c|c|c|c|}
\hline & & & Nhân tố & & \\
\hline & 1 & 2 & 3 & 4 & 5 \\
\hline CNSHI4 & 0,860 & & & & \\
\hline CNSHI3 & 0,848 & & & & \\
\hline CNSHI2 & 0,834 & & & & \\
\hline CNSHI1 & 0,824 & & & & \\
\hline CNSHI5 & 0,802 & & & & \\
\hline CNSTT2 & & 0,809 & & & \\
\hline CNSTT5 & & 0,803 & & & \\
\hline CNSTT4 & & 0,784 & & & \\
\hline CNSTT3 & & 0,761 & & & \\
\hline CNSTT1 & & 0,732 & & & \\
\hline CNRR4 & & & 0,789 & & \\
\hline CNRR3 & & & 0,745 & & \\
\hline CNRR5 & & & 0,740 & & \\
\hline CNRR2 & & & 0,722 & & \\
\hline CNRR1 & & & 0,666 & & \\
\hline CNDSD4 & & & & 0,813 & \\
\hline CNDSD2 & & & & 0,790 & \\
\hline CNDSD1 & & & & 0,763 & \\
\hline CNDSD3 & & & & 0,757 & \\
\hline CNCP3 & & & & & 0,835 \\
\hline CNCP2 & & & & & 0,831 \\
\hline CNCP1 & & & & & 0,779 \\
\hline
\end{tabular}


Nhân tố ảnh hưởng đến quyết định sử dụng dịch vụ Smart Banking- Nghiên cứu thực nghiệm tại BIDV- Chi nhánh Bắc Sài Gòn

Bảng 5. Ma trận hệ số tương quan Pearson

\begin{tabular}{cccccccc}
\hline \multirow{2}{*}{ QD } & & QD & CNSHI & CNDSD & CNSTT & CNCP & CNRR \\
\cline { 2 - 8 } & Pearson & 1 & $0,542^{* *}$ & $0,386^{* *}$ & $0,570^{* *}$ & $-0,388^{* *}$ & $-0,663^{* *}$ \\
\cline { 2 - 8 } & Sig. & & 0,000 & 0,000 & 0,000 & 0,000 & 0,000 \\
\hline \multirow{2}{*}{ CNSHI } & Pearson & $0,542^{* *}$ & 1 & $0,231^{* *}$ & $0,356^{* *}$ & $-0,164^{*}$ & $-0,390^{* *}$ \\
\cline { 2 - 8 } & Sig. & 0,000 & & 0,000 & 0,000 & 0,012 & 0,000 \\
\hline \multirow{2}{*}{ CNDSD } & Pearson & $0,386^{* *}$ & $0,231^{* *}$ & 1 & $0,179^{* *}$ & .012 & $-0,307^{* *}$ \\
\cline { 2 - 8 } & Sig. & 0,000 & 0,000 & & 0,006 & 0,857 & .000 \\
\hline \multirow{2}{*}{ CNSTT } & Pearson & $0,570^{* *}$ & $0,356^{* *}$ & $0,179^{* *}$ & 1 & $-0,214^{* *}$ & $-0,407^{* *}$ \\
\cline { 2 - 8 } & Sig. & 0,000 & 0,000 & 0,006 & & 0,001 & 0,000 \\
\hline \multirow{2}{*}{ CNCP } & Pearson & $-0,388^{* *}$ & $-0,164^{*}$ & 0,012 & $-0,214^{* *}$ & 1 & $0,363^{* *}$ \\
\cline { 2 - 8 } & Sig. & 0,000 & 0,012 & 0,857 & 0,001 & & 0,000 \\
\hline \multirow{2}{*}{ CNRR } & Pearson & $-0,663^{* *}$ & $-0,390^{* *}$ & $-0,307^{* *}$ & $-0,407^{* *}$ & $0,363^{* *}$ & 1 \\
\cline { 2 - 7 } & Sig. & 0,000 & 0,000 & 0,000 & 0,000 & 0,000 & \\
\hline
\end{tabular}

Ghi chú: ** Tương quan có ý nghĩa tại mức $1 \%$ (kiểm định 2 phía)

Nguồn: Phân tích tù dũ liệu thu thập được bằng SPSS 20

Pearson được sử dụng. Kết quả phân tích tương quan cho thấy tất cả các biến đều có tương quan với nhau tại mức ý nghĩa $1 \%$ (Bảng 5).

\subsection{Phân tích hồi quy}

Từ Bảng 6, kết quả ANOVA cho thấy trị thống kê $\mathrm{F}$ của mô hình $=84,343$ với mức ý nghĩa $1 \%(\operatorname{sig}=0,000)$, cho thấy mô hình hồi quy tuyến tính bội phù hợp với tập dữ liệu hay các biến độc lập có quan hệ tuyến tính với biến phụ thuộc và mô hình có thể sử dụng được. Mô hình có hệ số R2 hiệu chỉnh là 0,640 , hay $64 \%$ mức độ biến thiên Quyết định sử dung dịch vụ được giải thích bởi các biến độc lập.

Kết quả hồi quy cũng cho thấy: có 5 biến có ý nghĩa thống kê ở mức 1\% (Sig. $\leq$ $0,01)$, mô hình lý thuyết phù hợp với dữ liệu nghiên cứu và cả 05 giả thuyết nghiên cứu được chấp nhận. Phương trình hồi quy chưa chuẩn hóa có dạng:
$\mathrm{QD}=2,480-0,303 \times \mathrm{CNRR}+0,266 \times \mathrm{CNSTT}$ $+0,197 \times \mathrm{CNSHI}+0,174 \times \mathrm{CNDSD}-$ $0,156 \times \mathrm{CNCP}$

Trong việc dò tìm sự vi phạm các giả định hồi quy tuyến tính, Biểu đồ phân tán Scatterplot (Hình 2) cho thấy phần dư không thay đổi theo một trật tự nào đối với giá trị dự đoán, chúng phân tán ngẫu nhiên, giả thuyết về liên hệ tuyến tính không bị vi phạm. Hệ số tương quan hạng Spearman của giá trị tuyệt đối phần dư và các biến độc lập: giá trị Sig. của các hệ số tương quan với độ tin cậy $95 \%$ đều lớn hơn 0,05 , cho thấy phương sai của sai số không thay đổi, giả định không bị vi phạm. Biểu đồ Histogram (Hình 3) cho thấy phần dư có phân phối chuẩn với giá trị trung bình rất nhỏ gần bằng $0(\mathrm{Mean}=3.97 \mathrm{E}-15)$ và độ lệch chuẩn của nó gần bằng $1(\mathrm{SD}=0$, 0,989), Đồ thị P-P plot (Hình 4) biểu diễn các điểm quan sát thực tế tập trung khá sát đường chéo những giá trị kỳ vọng, có nghĩa là dữ liệu phần dư có phân phối chuẩn. Hệ 
Bảng 6. Hệ số hồi quy

\begin{tabular}{|c|c|c|c|c|c|c|c|c|}
\hline \multirow{2}{*}{\multicolumn{2}{|c|}{$\begin{array}{l}\text { Mô hình } \\
\text { B }\end{array}$}} & \multicolumn{2}{|c|}{$\begin{array}{c}\text { Hệ số hồi quy chưa } \\
\text { chuẩn hóa }\end{array}$} & \multirow[t]{2}{*}{$\begin{array}{l}\text { Hệ số hồi quy } \\
\text { chuẩn hóa }\end{array}$} & \multirow[t]{2}{*}{$\mathrm{T}$} & \multirow{2}{*}{$\begin{array}{c}\text { Sig. } \\
\text { Độ chấp } \\
\text { nhận }\end{array}$} & \multicolumn{2}{|c|}{$\begin{array}{l}\text { Thống kê đa } \\
\text { cộng tuyến }\end{array}$} \\
\hline & & Sai số chuẩn & Beta & & & & VIF & \\
\hline \multirow{6}{*}{1} & Hằng số & 2,480 & 0,282 & & 8,791 & ,000 & & \\
\hline & $\mathrm{CNSHI}$ & 0,197 & 0,036 & 0,242 & 5,482 & ,000 & 0,790 & 1,266 \\
\hline & CNDSD & 0,174 & 0,041 & 0,178 & 4,230 & ,000 & 0,871 & 1,148 \\
\hline & CNSTT & 0,266 & 0043 & 0,277 & 6,252 &, 000 & 0,782 & 1,278 \\
\hline & $\mathrm{CNCP}$ & $-0,156$ & 0,040 & $-0,168$ & $-3,932$ &, 000 & 0,844 & 1,185 \\
\hline & CNRR & $-0,303$ & 0,043 & $-0,340$ & $-7,017$ & ,000 & 0,653 & 1,530 \\
\hline
\end{tabular}

$R^{2}$ hiệu chỉnh: 0,640

Thống kê Durbin-Watson: 1,901

Thống kê $F$ (ANOVA): 84,343

Mức ý nghĩa (Sig. của ANOVA): 0,000

Nguồn: Phân tích tù̀ dũ liệu thu thập được bằng SPSS 20

Hình 2. Biểu đồ phân tán giữa hai biến giá trị phần dư và giá trị dụ̣ đoán

Scatterplot

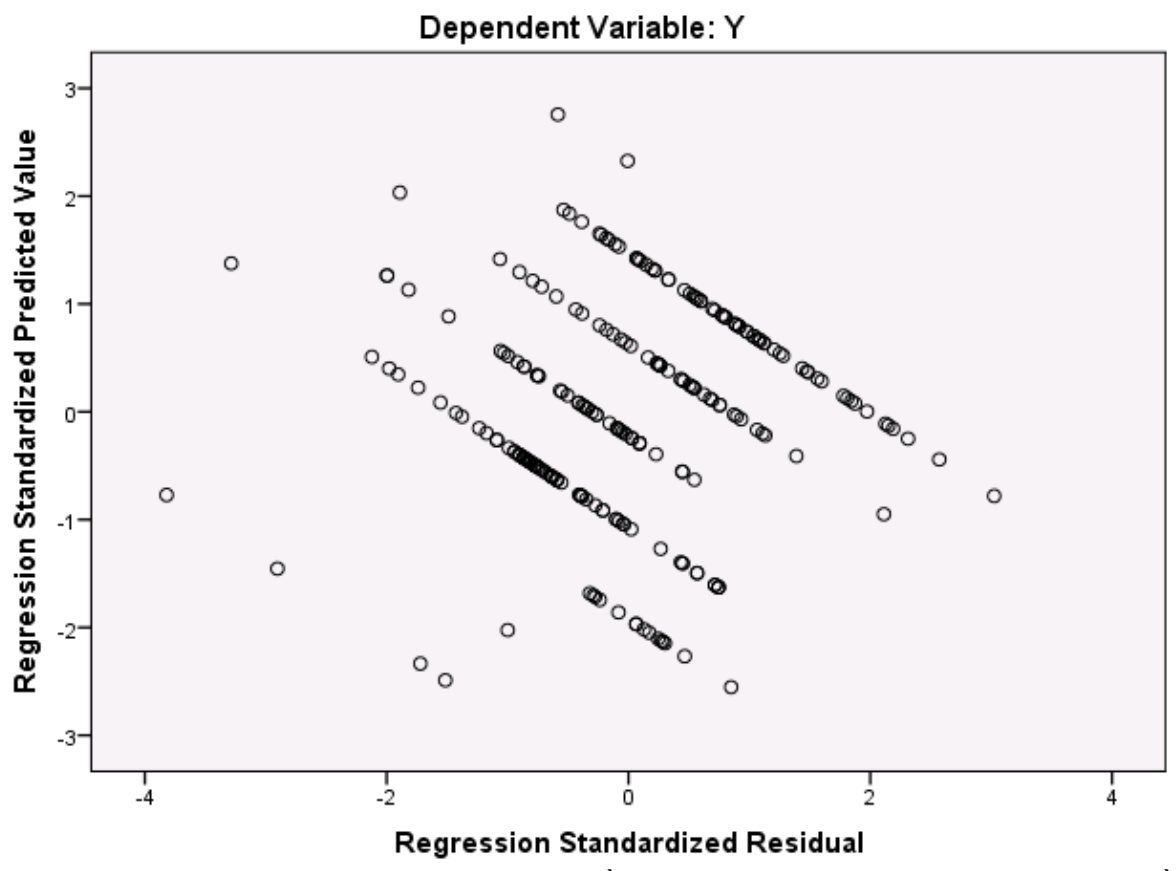

Nguồn: Phân tích tù dũ liệu thu thập được bằng SPSS 20

số $1<$ Durbin-Watson= $1,901<3$ là thỏa mãn điều kiện, hệ số phóng đại phương sai $\mathrm{VIF}<10$ cho thấy các biến độc lập không có quan hệ chặt chẽ với nhau nên không xảy ra hiện tượng đa cộng tuyến. Như vậy, mô hình hồi quy tuyến tính được xây dựng theo phương trình trên không vi phạm các giả định hồi quy.

\subsection{Kiểm định sụ khác biệt}

Kiểm định t-test cho thấy không có sự khác 
biệt về Quyết định sử dụng dịch vụ giữa nam và nữ. Kiểm định ANNOVA cho thấy không có sự khác biệt về Quyết định sử dụng dịch vụ giữa các nhóm khách hàng về thời gian sử dụng dịch vụ BIDV, độ tuổi, nghề nghiệp, thu nhập, trình độ học vấn khác nhau.

\subsection{So sánh kết quả nghiên cúvu}

Trong nghiên cứu này, các nhân tố và chiều tác động đến quyết định sử dụng dịch vụ Smart Banking giảm dần theo thứ tự sau: (1) Cảm nhận rủi ro (tác động ngược chiều), (2) Cảm nhận sự tin tưởng (tác động cùng chiều), (3) Cảm nhận sự hữu ích (tác động cùng chiều), (4) Cảm nhận dễ sử dụng (tác động cùng chiều) và (5) Cảm nhận chi phí (tác động ngược chiều). Kết quả nghiên cứu đúng như 5 giả thiết (từ $\mathrm{H} 1$ đến $\mathrm{H} 5$ ) đã đặt ra về chiều tác động của các nhân tố. Về mức độ tác động và so sánh kết quả nghiên cứu thể hiện qua Bảng 7 .

\section{Kết luận và hàm ý quản trị}

\subsection{Kết luận}

Qua quá trình nghiên cứu, nhóm tác giả đã sử dụng phương pháp nghiên cứu định lượng phù hợp, xử lý số liệu bằng phương tiện thống kê để có thể xác định được 05 nhân tố tác động đến Quyết định sử dụng dịch vụ Smart Banking tại BIDV- BSG, sắp xếp theo thứ tự giảm dần về mức độ tác động: (1) Cảm nhận rủi ro (tác động ngược chiều), (2) Cảm nhận sự tin tưởng (tác động
Hình 3. Biểu đồ Histogam Histogram

Dependent Variable: $Y$

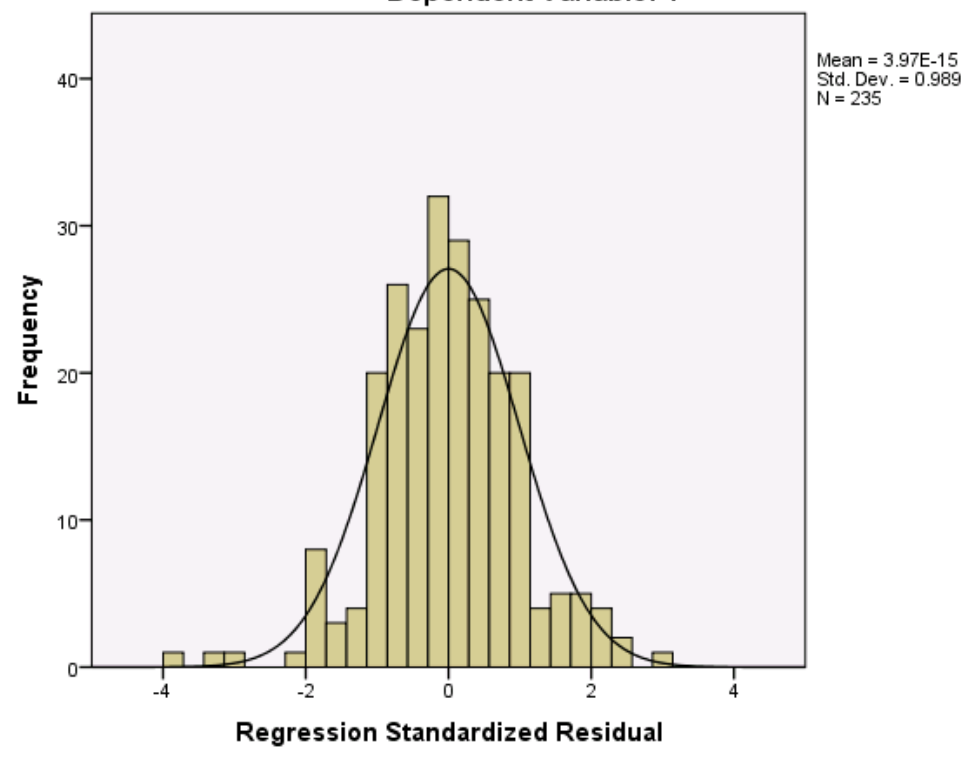

Nguồn: Phân tích tù dũ liệu thu thập được bằng SPSS 20

Hình 4. Đồ thị P-P plot

Normal P-P Plot of Regression Standardized Residual

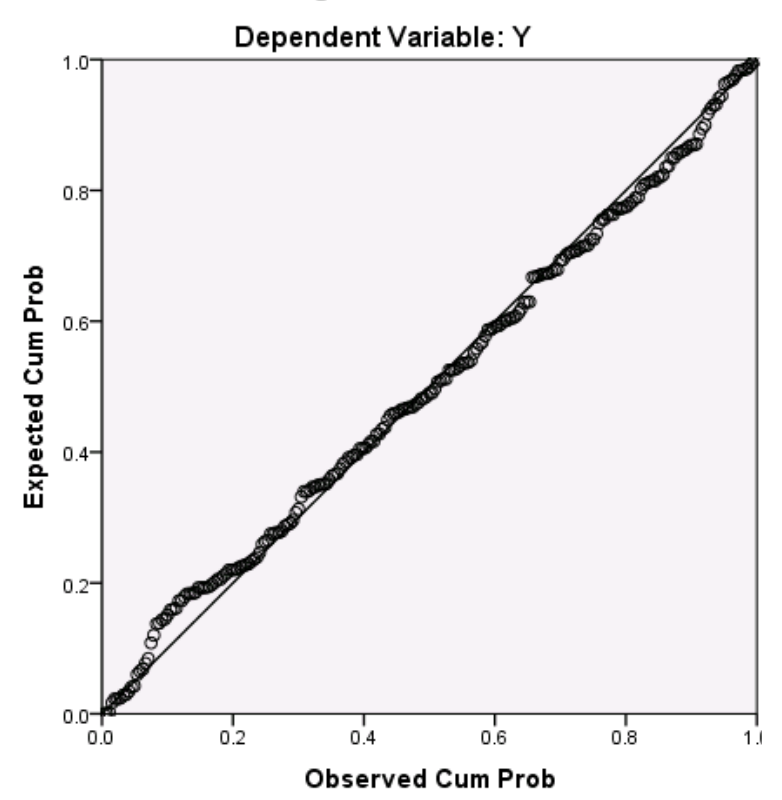

Nguồn: Phân tích tì̀ dũ liệu thu thập được bằng SPSS 20

cùng chiều), (3) Cảm nhận sự hữu ích (tác động cùng chiều), (4) Cảm nhận dễ sử dụng (tác động cùng chiều) và (5) Cảm nhận chi phí (tác động ngược chiều). Đây là cơ sở để 
các nhà quản trị BIDV BSG lưu ý khi xây dựng chiến lược khách hàng.

\section{2. Đề xuất hàm ý quản trị}

\section{Cảm nhận rủi ro}

Khách hàng đánh giá khá thấp cảm nhận rủi ro với giá trị trung bình là 2,69 , đồng nghĩa với việc khách hàng tin tưởng vào dịch vụ này cao. Khách hàng đã và đang sử dụng dịch vu Smart Banking rất quan tâm đến việc bảo mật thông tin cá nhân, lỗi hệ thống và vấn đề bị kẻ xấu tấn công. BIDV BSG cần tiếp tục duy trì phát huy để khách hàng ngày càng yên tâm, tin tưởng nhiều hơn nữa. BIDV BSG cần xây dựng đội ngũ công nghệ thông tin mạnh, có kiến thức về công nghệ và am hiểu về ngành Ngân hàng, kết hợp với việc hiểu tâm lý khách hàng sẽ giúp các dịch vụ Smart Banking ngày càng phát triển mạnh hơn. BIDV BSG cần nghiên cứu đưa ra dịch vụ có độ phức tạp phù hợp, đơn giản nhưng đảm bảo tính bảo mật cao.

\section{Cảm nhận sụ tin tưởng}

Cảm nhận sự tin tưởng được khách hàng đánh giá tương đối cao với mức trung bình
3,38. BIDV BSG cần quan tâm đến những ứng dụng công nghệ và mong muốn những công nghệ này mang đến sự an toàn bảo mật cho khách hàng. Phần lớn khách hàng 10 lắng về tiết lộ những tin giao dịch tài chính, vẫn chưa có niềm tin về tính trung thực của ngân hàng. Vì vậy, để khuyến khách hàng giao dịch thông qua Smart Banking, BIDV BSG nên đưa ra những cam kết mạnh mẽ. Nhân viên giao dịch khi tư vấn cho khách hàng phải tận tình, rõ ràng và luôn thể hiện tính an toàn trong giao dịch.

\section{Cảm nhận sụ̂ hũu ích}

Khách hàng đánh giá nhân tố này khá cao với giá trị trung bình là 3,36. BIDV nên phát huy những thế mạnh mà dịch vụ Smart Banking mang đến cho khách hàng: sự nhanh chóng, tiện lợi, tiết kiệm chi phí hơn giao dịch trực tiếp. BIDV BSG phải chú tâm, nhiệt tình hơn đối với công nghệ giao dịch qua Smart Banking, luôn có đội ngũ kiểm tra những lỗi hệ thống báo về và nhanh chóng sửa những lỗi đó. Đồng thời, cần luôn học hỏi công nghệ này trên thế giới và nghiên cứu ứng dụng tại Việt Nam.

\section{Cảm nhận dễ sử dụng}

Bảng 7. So sánh với các nghiên cứu trước về mức độ ảnh hưởng của các nhân tố đến quyết định sử dụng dịch vụ

\begin{tabular}{|c|c|c|c|c|c|}
\hline STT & $\begin{array}{l}\text { Shanmugam và } \\
\text { cộng sự (2014) }\end{array}$ & $\begin{array}{l}\text { Al-Jabri và } \\
\text { Sohail (2012) }\end{array}$ & $\begin{array}{l}\text { Yoon và } \\
\text { Occena (2015) }\end{array}$ & $\begin{array}{l}\text { Lê Tô Minh Tân } \\
\text { (2013) }\end{array}$ & $\begin{array}{l}\text { Nghiên cứu của } \\
\text { Tác giả }\end{array}$ \\
\hline 1 & $\begin{array}{l}\text { Nhâan thức sự } \\
\text { hữu ích }\end{array}$ & Nhận thức rủi ro & $\begin{array}{l}\text { Nhâan thức sự an } \\
\text { toàn }\end{array}$ & Cảm nhận rủi ro & Cảm nhận rủi ro \\
\hline 2 & $\begin{array}{l}\text { Nhận thức sự tín } \\
\text { nhiệm }\end{array}$ & Tính khả thi & $\begin{array}{l}\text { Nhận thức sự } \\
\text { hữu ích }\end{array}$ & $\begin{array}{l}\text { Cảm nhận dễ sử } \\
\text { dụng }\end{array}$ & $\begin{array}{l}\text { Cảm nhận sự tin } \\
\text { tưởng }\end{array}$ \\
\hline 3 & $\begin{array}{l}\text { Nhận thức lợi } \\
\text { ích }\end{array}$ & Sự phức tạp & $\begin{array}{l}\text { Nhận thức dễ sử } \\
\text { dụng }\end{array}$ & $\begin{array}{l}\text { Cảm nhận sự } \\
\text { hữu ích }\end{array}$ & $\begin{array}{l}\text { Cảm nhận sự } \\
\text { hữu ích }\end{array}$ \\
\hline 4 & $\begin{array}{l}\text { Nhận thức dễ sử } \\
\text { dụng }\end{array}$ & Sự tương thích & & & $\begin{array}{l}\text { Cảm nhận dễ sử } \\
\text { dụng }\end{array}$ \\
\hline 5 & $\begin{array}{l}\text { Nhận thức về } \\
\text { chi phí tài chính }\end{array}$ & Sự thuận tiện & & & $\begin{array}{l}\text { Cảm nhận chi } \\
\text { phí }\end{array}$ \\
\hline 6 & & Tính ưu việt & & & \\
\hline
\end{tabular}

Nguồn: Tổng hợp của nhóm nghiên cưu 
Khách hàng đánh giá nhân tố cảm nhận dễ sử dụng ở mức trung bình (mean) là 3,37. BIDV BSG cần luôn tìm cách để khách hàng dễ dàng tiếp cận với dịch vụ Smart Banking nhanh nhất. BIDV BSG cần xây dựng những chương trình nhằm khuyến khích khách hàng sử dụng dịch vụ Smart Banking như hỗ trợ cho khách hàng mới chuyển tiền 10 lần không mất phí hoặc hỗ trợ đường dây nóng cho khách hàng. Đồng thời, có thể gửi email hoặc gửi cho khách giấy hướng dẫn thao tác thực hiện giao dịch.

\section{Cảm nhận chi phí}

Cảm nhận chi phí được khách hàng cho rằng chi phí thấp với mean là 2,53 . BIDV $\mathrm{BSG}$ cần duy trì và phát huy điểm mạnh về các chi phí kết nối mạng và phí giao dịch vẫn còn rẻ hơn nhiều so với việc khách hàng tự bỏ thời gian, chi phí đến ngân hàng để giao dịch. Ngoài ra, BIDV BSG cần truyền thông, giới thiệu mạnh mẽ thêm để khách hàng hoàn toàn yên tâm về vấn đề cài đặt ứng dụng Smart banking trên điện thoại.

\subsection{Hạn chế và hướng nghiên cứu tiếp theo}

Nghiên cứu vẫn còn một số hạn chế nhất định: (1) Nghiên cứu được thực hiện theo phương pháp lấy mẫu thuận tiện, phi xác suất nên chưa có tính đại diện cao, các nghiên cứu tiếp theo có thể cân nhắc các phương pháp chọn mẫu xác suất để có tính đại diện cao hơn; (2) $\mathrm{R}^{2}$ hiệu chỉnh của nghiên cứu là 0,640 , cho thấy có thể còn nhiều nhân tố khác ảnh hưởng đến quyết định sử dụng dịch vụ Smart Banking tại BIDV BSG, các nghiên cứu tiếp theo cần tìm hiểu bổ sung để kết quả ngày càng hoàn thiện hơn

\section{Tài liệu tham khảo}

1. Al-Jabri Ibrahim M. \& Sohail M. Sadiq. 2012, "Mobile Banking Adoption: Application of Diffusion of Innovation Theory”, Journal of Electronic Commerce Research, Vol. 13 No. 4, pp. 379-391.

2. Bui Nhat Vuong- Ha Nam Khanh Giao, 2019. "The impact of brand globalness on consumers purchase intention and the moderating role of consumer ethnocentrism an eveidence from Vietnam". Journal of International Marketing, Vol. 32, No. 1, pp. 47-68. DOI: 10.1080/08961530.2019.1619115

3. Davis, F. D. 1989, "Perceived Usefulness Perceived Ease of Use, and User Acceptance of Information Technology”, Mis Quarterly, Vol. 13, pp. 319- 340.

4. Davis, F. D., Bagozzi, R. P. \& Warshaw, P. R. 1989, "User Acceptance of Computer-Technology-a Comparison of 2 Theoretical-Models”, Management Science, Vol. 35, pp. 982-1003.

5. Foon, Y. S. \& Fah, B. C. Y. 2011, "Internet Banking Adoption in Kuala Lumpur: An Application of UTAUT Model", International Journal of Bussiness and Management, Vol. 6, pp. 161- 167.

6. Ha Nam Khanh Giao - Bui Nhat Vuong - Tran Nhu Quan, 2019. "The influence of website quality on consumer's e-loyalty through the mediating role of e-trust, esatisfaction, and perceived enjoyment: An evidence from online shopping in Vietnam”. Uncertain Supply Chain Management, Vol. 8, No. 2, pp. 351-370. DOI: 10.5267/j. uscm.2019.11.004.http://www.growingscience.com/uscm/Vol8/uscm_2019_42.pdf.

7. Hà Nam Khánh Giao. 2004. "Marketing Dịch vu- Mô hình 5 Khoảng cách Chất lương Dịch vụ”. Nhà Xuất bản Thống kê.

8. Hà Nam Khánh Giao- Bùi Nhất Vưong. 2019. "Giáo trình cao học-Phuoong pháp nghiên cúu khoa học trong kinh doanh- Cập nhật SmartPLS”. Nhà xuất bản Tài chính. Hà Nội. DOI: 10.31219/osf.io/hbj3k.

9. Hà Nam Khánh Giao, 2018. Sách chuyên khảo "Đo lường chất luợng dịch vu tại Việt Nam- nhìn tù góc độ khách hàng”. Nhà xuất bản Tài chính. Hà Nội. DOI: 10.31219/osf.io/cqh68.

10. Hà Nam Khánh Giao, Ao Thu Hoài, Phạm Quang Vinh. 2019. "Quản trị Kinh doanh Dịch vụ- Tù Góc nhìn 
Marketing”. Nhà xuất bản Truyền thông Thông tin Hà Nội. DOI: 10.31219/osf.io/98hrd.

11. Hughes, A. 2018. "Market Driven Political Advertising: Social, Digital and Mobile Marketing”. Springer.

12. Jeong, B. K\& Yoon, T. E. 2013, “An Empirical Investigation on Consumer Acceptance of Mobile Banking Service”, Business and Management Research, Vol. 2, pp. 31- 40.

13. Lê Tô Minh Tân. 2013, "Giải pháp phát triển dịch vụ ngân hàng trục tuyến ở Thừa Thiên Huế", Đề tài nghiên cứu khoa họ cấp co sỏ, Truò̀ng Đại học Kinh tế Huế.

14. Luarn, P., and Lin, H.H. (2005), "Toward an understanding of the behavioural intention to use mobile banking", Computers in Human Behaviour, Vol. 21, pp.873-891. ttp://dx.doi.org/10.1016/j.chb.2004.03.003

15. Nguyễn Thế Phuoong, 2014, Các nhân tố ảnh hưởng đến quyết định sủ dụng dịch vu mobile banking của khách hàng cá nhân tại Ngân hàng Vietcombank, luận văn Thạc sỹ kinh tế, Trương Đại học tài chính-Marketing.

16. Rogers, E. M. 1995, Diffusion of Innovations, 4th edn, Free Press, New York.

17. Shanmugam Arunagiri, Savarimuthu Michael Thaz, Wen Teoh Chai. 2014, "Factors Affecting Malaysian

Behavioral Intention to Use Mobile Banking With Mediating Effects of Attitude”, Academic Research International,

Vol. 5 No. 2, pp. 236-253.

18. TTBC số 03/2019: BIDV - "Ngân hàng Bán lẻ tốt nhất Việt Nam" 5 năm liên tiếp. https://www.bidv.com.vn/bidv/ tin-tuc/thong-tin-bao-chi/ttbc+so+03-2019+-+giai+thuong+nhbl+tot+nhat+2019. Truy câp ngày 20/01/2020.

19. Venkatesh, V., Morris, M. G., Davis, G. B. \& Davis, F. D. 2003, "User acceptance of information technology:

Toward a unified view", MIS Quarterly, Vol. 27, pp. 425-478.

20. Wu, J.-H. \& Wang, S.-C. 2005, "What drives mobile commerce? An ampirical evaluation of the revised technology acceptance model”, Information \& Management, Vol. 42, pp. 719- 729.

21. Yoon, H. S., \& Occeña, L. G. 2015, "Influencing factors of trust in consumer-to-consumer electronic commerce with gender and age”, International Journal of Information Management, Vol. 35 No.3, pp. 352-363.

22. Yu, C.-S. 2012, "Factors Affecting Individuals to Adopt Mobile Banking”, Journal of Electronic Commerce Research, Vol. 13, pp. 104- 121.

23. Xuân Lộc-Hương Giang (2019). Bộ Thông tin-Truyền thông báo cáo so kết 6 tháng đầu năm và phưong huóng nhiệm vu 6 tháng cuối năm 2019. http://www.mic.gov.vn/Pages/TinTuc/139313/Bo-TT-TT-so-ket-cong-tac-6-thangdau-nam-va-phuong-huong-nhiem-vu-6-thang-cuoi-nam-2019.html. 